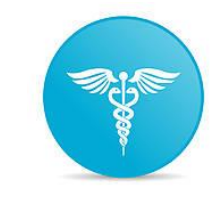

International Journal of Advances in Pharmacy and Biotechnology

Vol.1, Issue-2, 2015, 19-24

I J A P B

Review Article

Open Access

\title{
GOLD NANOPARTICLES IN CANCER THERAPY
}

\author{
M. Deepa*, M. Surekha, V. Thushara Bindu, Deepti, K. Bhargavi, S. Deva sree \\ ${ }^{*}$ P. Rami Reddy Memorial College of Pharmacy, Kadapa, A.P.-516003, India. \\ *Corresponding author e-mail: venkatdeepa19@gmail.com
}

\begin{abstract}
Received: 21 October 2015
Revised: 05 November 2015

Accepted: 09 November 2015

ABSTRACT:

Gold nanoparticles have many properties that are attractive for the use in the cancer therapy. They are small and widely penetrate into the body, preferentially accumulating at the tumor sites owing to EPR effect .They can bind many proteins and drugs and can be actively targeted to cancer cells over expressing cell surface receptors. Gold nanoparticle have high atomic number, which leads to greater absorption of kilovoltage Xrays and provide greater contrasts than standard agents. Gold nanoparticles have been shown to cause radio sensitization at kilovoltage and megavoltage photon energies. The factors that affect gold nanoparticle pharmacokinetics, bio-distribution and in-vivo toxicity need to be clarified. Targeted gold nanoparticle need to exit tumour vasculature, cross the tumour intersitium, enter the cells and potentially exit lysosomes to be effective in-vivo. A standard approach for physicochemical characterization and pre-clinical testing needs to be implemented and this process is being aided by the NLC. With intense global interest in nanotechnology and particularly in nanomedicine.
\end{abstract}

Key words: Goldnanoparticles, EPR effect, Tumour, PEGylation, Targeting.

\section{INTRODUCTION Nanoparticles[ ${ }^{1]}$}

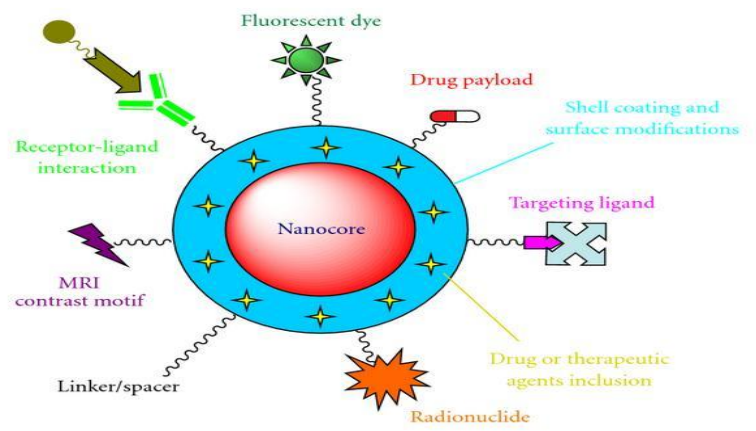

Fig. 1 Structure of Nanoparticles

Nanoparticles have several biomedical and industrial applications in diagnosis of disease targeted chemotherapy and in drug delivery. Multi functionality and sub micronic size is the main characteristics of nanoparticles can be integrated with ligands, imaging labels, therapeutic agents and other functionalities for site specific drug delivery and cellular uptake. In the present review we are discussing the application (Fig. 1) and synthesis of gold nanoparticles which is the most studied among all metallonanoparticles.

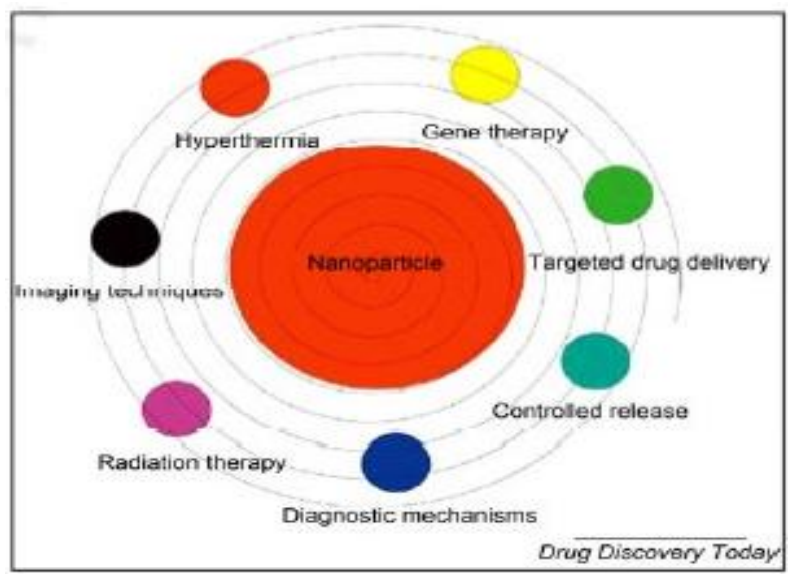

Fig. 2 Uses of Nanoparticles

Various anticancer drugs are available but these are cause the necrosis of cancerous cell as well as normal cells. But gold nanoparticles cause the necrosis of only cancer cells. These are targeted drug delivery systems which are smaller than human cells so can easily penetrate the tumour and destroy the cancerous cell. Various anti cancer drugs 
conjugated with gold nanoparticles result in increased efficiency of anticancer drug. Gold nanoparticles are beneficial for chemotherapy and also for diagnosis of cancer.

\section{History of Nanoparticles ${ }^{2]}$}

The historical development of nanoparticles starting with Paul Ehrlich and then first attempts by Ursula Scheffel and collegues and the extensive work by the group of professor Peter Speiser at the ETH Zurich in the late 1960s and early 1970s are described from a personal point of view. Nanoparticles for the delivery of drugs across the blood brain barrier (BBB) and PEGylated nanoparticles with a prolonged blood circulation time.

\section{Types of Nanoparticles}

1. Magnetic Nanoparticles

2. Metallic Nanoparticles

\section{Magnetic Nanoparticles}

Magnetic nanoparticles are a class of nanoparticles which can be manipulated using magnetic field, because they possess attractive properties which could see potential use in catalyst, biomedicine, magnetic resonance imaging, data storage. Such particles containing iron, nickel and cobalt and their chemical compounds. While nanoparticles are smaller than 1 micrometer in diameter (typically 5-500 nanometers), the larger microbeads are 0.5-500 micrometer in diameter.

Types of Magnetic Nanoparticles

1. Oxides: Ferrite

2. Metallic

3. Metallic with a shell

\section{Metallic Nanoparticles}

Today these materials can be synthesized and modified with various chemical functional groups which allow them to be conjugated with antibodies, ligands. In addition to various imaging techniques in tandem newer multifunctional nanoshells ${ }^{[3]}$ and nanocages have been developed. Thus in this review article, we aim to provide an introduction to magnetic nanoparticles, gold nanoparticles, nanoshells and nanocages, and silver particles followed by their synthesis, physicochemical properties.

\section{Types of Metallic Nanoparticles}

1. Iron oxide nanoparticle

2. Nanoshell \& nanocages

3. Silver nanoparticles

4. Gold nanoparticles

\section{GOLD NANOPARTICLES}
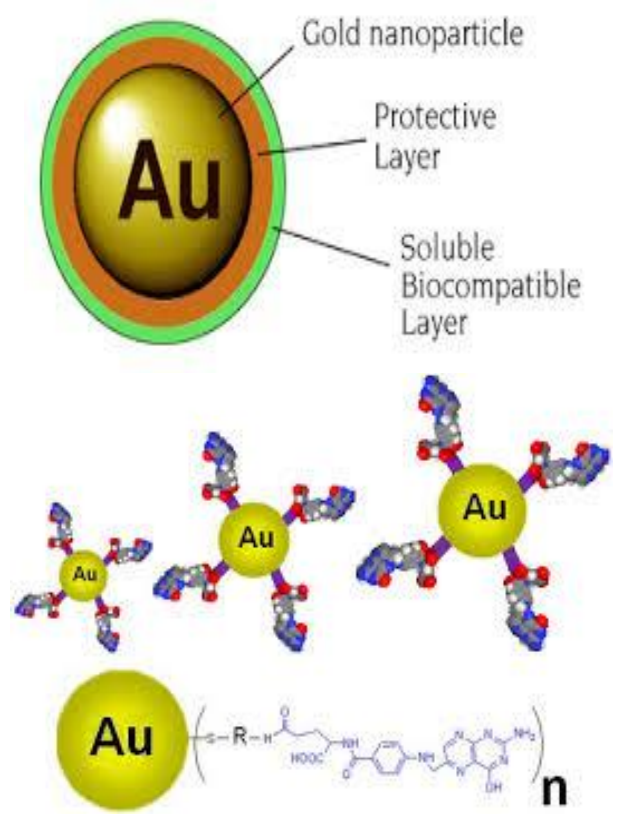

Fig. 3 Structure of gold nanoparticles

Gold nanoparticles have gained increasing interest due to their special features, such as an unusual optical and electronic properties, high stability and biological compatability, controllable morphology and size dispersion and easy surface functionalization. In typical synthesis ${ }^{[4]}$ AuNps are produced by reduction of gold salts in appropriate solvents.

After synthesis the stabilising agent surrounding the AuNPs can be replaced by other molecules by ligand exchange reactions. Functionalisation of AuNPs makes it possible to adjust the surface properties and attach different kinds of molecules to the particles.

AuNPs have shown the great potential applications in the fields of chemistry, physics, biology and medicine. The exciting finding is that AuNPs have shown a great potential in therapies for HIV. They have stopped HIV from infecting human white blood cells.

\section{Characterstics $^{[5]}$ of Gold Nanoparticles} (Fig. 2)

- Gold nanoparticles are chemicaly inert. 
- These have greater biological compatability.

- They have stability due to the gold sulphur bonds.

- Their photo physical properties can be exploited for drug release at remote place.

- Gold nanoparticles provide microscopic probes for study of cancer cells.

Types of Gold Nanoparticles

1. Gold nanorods

2. Gold nanoshells

3. Gold nanocages

4. Gold nanosphere

5. SERS nanoparticles

\section{Gold Nanorods ${ }^{[6]}$}

These are synthesized by template method. These are prepared by electrochemical deposition of gold within the pores of nanoporous polycarbonate template membranes.

\section{Gold Nanoshells}

Surface plasmon resonance peaks (ranging from visible to near i.r region) is used for the designing and fabrication of gold nanoshells. The core of gold nanoshells is made up of silica and outer surface is made up of gold.

\section{Gold Nanocages}

Through galvanic replacement reaction between truncated silver nanotubes and aqueos $\mathrm{HAuCl}_{4}$, gold nanocage is synthesized.

\section{Sers Nanoparticles ${ }^{[7]}$}

Sers is an optical technique like flouresence and chemiluminescence having better sensitivity, high levels of multiplexing, robustness and greater performance in lood and biological.

\section{Solid Nanosphere}

These are synthesized by reduction of an aqueous haucl4, by using citrate as reducing agent. Through citrates/gold ratio the size of nanospheres can be affected by thiol/gold molar ratios.

\section{Synthesis ${ }^{[8]}$ of Gold Nanoparticles}

1. Intially prepared aquaregia solution which contains 3 parts of HCL and 1 part of $\mathrm{HNO}_{3}$ which is highly corrosive and oxidizing agent.

2. Then use chloroauric acid as reactant and sodium borohydride as reducing agent. NaBH solution+Deionised water.

3. The change in colour of goldfrom yellow to ruby red indicates the preparation of gold nanoparticles.

4. Gold nanoparticles of 1-2 $\mathrm{nm}$ can be prepared by using diborane as reducing agent with phosphine as stabilising agent to prevent agglomeration. Gold nanoparticles of 10-150 $\mathrm{nm}$ can be prepared by using sodium citrate as reducing gent in water and citrate as stabilising agent.

5. After synthesis the other molecules replace the capping agents by ligand exchange reaction

\section{Functionalisation of Gold Nanoparticles}

Mono layer protected clusters can be prepared by one-pot protocol drug for cancerous cells. Mixed mono layer protected cluster can be prepared directly or by murray through place exchange reaction of thiol.

Coumarin, a fluorescent dye when is conjugated with poly ethylene glycol at one end and at the other end gold nanoparticles. The effect of coumarin-PGE-thiol[9] is increased. So due to PEG the fluorescent receptor quenching effect of gold nanoparticles is decreased.

So through PEG spacer the gold nanoparticles, in the aqeous disruption of gold nanoparticles coumarin-PGE thiol are added for conjugation. There is a covalent interaction.

Invivo targeting[10] using gold nanoparticles. It can be done by two approaches.

1. Active targeting

2. Passive targeting

Active targeting depends on recognition of ligand on the gold nanoparticles surface by cell surface receptors.

Passive targeting depends on the extravasations of vectors through leaky blood vessels in unhealthy tissue.

- Gold nanoparticles are used to identify the protein interaction under the study of immunohistochemistry [11].

- Gold nanorods can be used to detect the cancer stem cells. 
- These are used to detect the aminoglycoside antibiotics like streptomycin,gentamycin,neomycin.

- These are used as lab tracer to detect the pressure of DNA in the sample. So these are used in fingerprinting.

\section{Applications of Nanoparticles[12]}

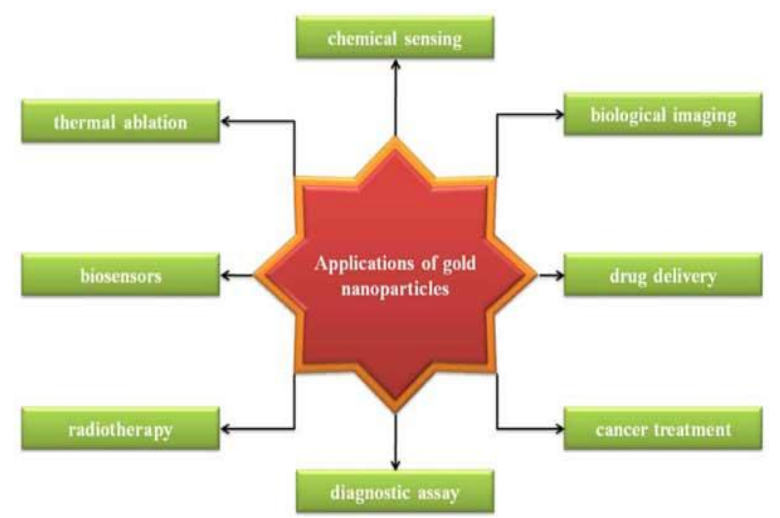

Fig. 4 Flow Chart of application of nanoparticles

1. Delivery of proteins, peptides and nucleic acid

2. Delivery of drugs

3. Gold nanoparticles in microbiology and science

4. Gold nanoparticles in diagnostic

5. Goldnanoparticles in therapy

6. Gold nanoparticles as drug carriers

7. Immunological properties of gold particles

8. Gold nanoparteicles in clinical trails

Delivery of Proteins ${ }^{[13]}$, Peptides and Nucleic Acid

These can be employed as a carrier for delivery of peptides, proteins and nucleic acids like DNA due to their tunable size.

GNPS are funtionalised with cationic quartenary ammonium groups can bind DNA plasmid through electrostatic interaction and protect DNA from enzymatic digestion. GNPs can work as a carrier for peptides and proteins; have reported that the Trans mucosal delivery of insulin is enhanced.

\section{Delivery ${ }^{[14]}$ of Drugs:}

GNPs cause local heating when irradiated with light in 800 to $1200 \mathrm{~nm}$. They cause the photo thermal destruction of tumours. GNPs are used to identify protein interaction under the study of immunohistochemistry. These are used as a lab tracer to detect the presence of DNA in sample. They are used for the detection of aminoglycosides antibiotics like streptomycin, gentamycin and neomycin. Gold Nanorods can used to detect the cancer stem cells.

\section{Gold Nanoparticles in Microbiology and Science}

The application of GNPs conjugates in biochemical diagnostics [15] and analytics, photothermal and photodynamic therapies, as a carrier for delivering target molecules and on the immunological and toxicological properties.

Gold Nanoparticles in Diagnostics:

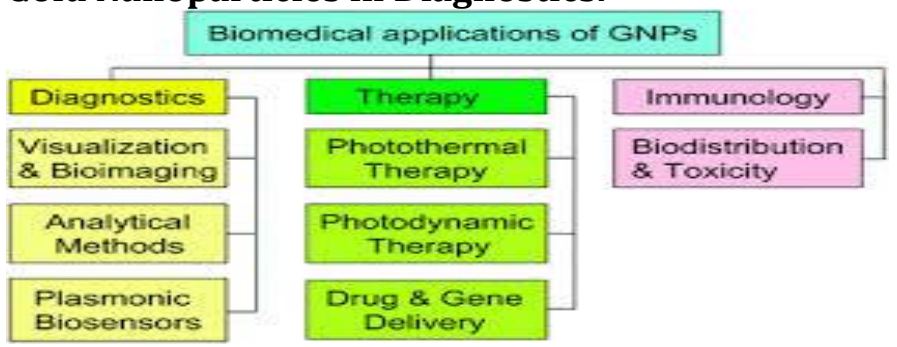

Fig. 5 Flow chart of Biomedical Application of Gold Nanoparticles

\section{Visualisation and Bioimaging}

GNPs are used in resonance scattering dark field microscopy for the detection of microbial cells and their metabolites, the bioimaging of tumour cells and for the detection of receptors on their surface, for the study of endocytosis.

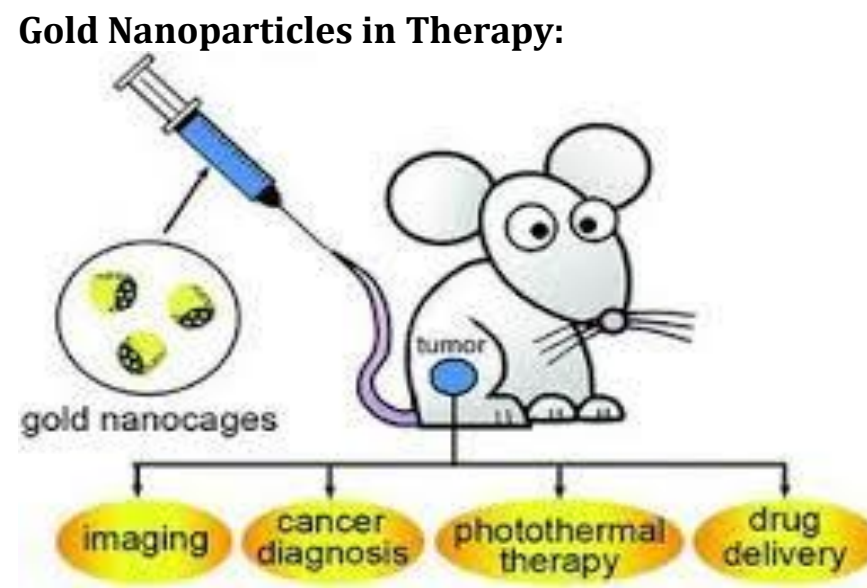

Fig. 6 Diagramatic Representation of Gold Nanoparticles in Therapy 


\section{Photothermal Therapy using Gnps}

Photothermal cell damage is a promising direction in both tumour therapy and therapy of infectious diseases.

\section{Photo Dynamic Therapy using Gnps ${ }^{[16]}$}

It is applied in therapy of oncological diseases, certain dermal or infectious diseases and is based on use of photo sensitizers. The agents for photo dynamic therapy (PDT) can selectively accumulate in the tumour or other target tissues. The main drawback of PDT is that photo sensitizers remain in organism for a long period of time; as a result, the patient's tissues remain highly sensitive to light.

\section{Gold Nanoparticles as Drug Carriers}

The targeted delivery of drugs is one of the most promising and actively developing directions in the medicinal use of GNPs. Antitumour agents and antibiotics are the most popular objects of target delivery. The options of using GNP conjugated with the following antitumour agents were proposed: paclitaxel, methotrexate, sulfonamide, platinum complexes, hemcytabin etc.

\section{Immunological Properties of Gold Nanoparticles}

The physic chemical immunity theory, postulates ${ }^{[17]}$ that immunogenicity and antigenic specificity depends prominently on physic chemical properties of the compounds and on their colloidal state .Unfortunately with the development of immunology and negation of many postulates, interest towards the immunological properties of colloids has abated.

\section{Gold Nanoparticle Clinical Trails}

The national cancer institute, the US food and drug administration, the national institute of standards and technologies, performs and standardises the preclinical characterisation of nanoparticles intended for cancer therapy .Previous clinical trials of TNS-alpha demonstrated dose limiting toxicity of hypotension and nausea at 225 micrograms. Dose levels of 50-60 micrograms TNF were administered with no dose limiting toxicity observed.

\section{Advantages and Disadvantages of Gold Nanoparticles Advantages}

1. Simple diagnosis

2. Less invasive

3. Diagnosis of oral cancer

4. Non-toxic to human beings

5. Gold is virtually indestructible, it lasts forever.

6. It is inert to all chemical, encounters in the body.

\section{Disadvantages}

1. Biocompatability

2. Acute and chronic toxicity

3. Ability to escape reticulo endothelial system need further researches.

\section{CONCLUSION}

The use of Gold nanoparticles in cancer offers exiting possibilities such as destroying cancer tumours with minimal damage to healthy tissues and organs as well as the detection and elimination of cancer cells before they form tumours.

Many efforts to improve cancer treatment through nanoparticles are at the research and developing stage. It is possible that these efforts will result in cancer becoming being nearly eliminated smallpox in the last century.

Gold nanoparticles have been investigated in diverse areas such as in vitro assays, in vitro and in vivo imaging, and cancer therapy and drug delivery.

\section{REFERENCES}

1. Anvika Tomar and Garima Garg. Short Review on Application of Gold Nanoparticles. Global Journal of Pharmacology, 2013; 7(1): 34-38.

2. Jorg Kreuter. Nanoparticles-a historical prospective. International Journal of Pharmaceutics, 2006; 331(2007): 1-10.

3. Nie S and Emory SR. Probing Single Molecules and Single Nanoparticles by Surface-Enhanced Raman Scattering. Science, 1997; 275: 1102-1106.

4. Rosi NL. Giljohann DA, Thaxton CS, Lytton-Jean AKR, Han MS and Mirkin CA. Oligonucleotide-Modified Gold Nanoparticles Fr Intracellular Gene 
Regulation. Science, 2006; 312: 10271030.

5. Sperling RA, Gil PR, Zhang F, Zanella M and Parak WJ. Biological Applications of Gold Nanaoparticles. Chem. Soc Rev, 2008; 37: 1896-1908.

6. Grzelczak M, Perez-Juste J, Mulvaney P and Liz-Marzan LM. Shape Control in Gold Nanoparticle Synthesis. Chem. Soc. Rev, 2008; 37: 1783-1791.

7. Templeton AC, Wuelfing WP and Murray RW. Monolayer-Protected Cluster Molecules. Acc. Chem. Res, 2000; 33: 2736.

8. Shankar SS, Rai A, Ankamwar B, Singh A, Ahmad A and Sastry M. Biological Synthesis of Triangular Gold Nanoprisms. Nature Materials, 2004; 3: 482-488.

9. McGilvray KL, Decan MR, Wang D and Scaiano JC. Facile Photochemical Synthesis of Unprotected Aqueous Gold Nanoparticles. J. Am. Chem. Soc. 2006; 128: 15980-81.

10. Lofton C and Sigmund W. Mechanisms Controlling Crystal Habits of Gold and Silver Colloids. Adv. Funct. Mate. 2005; 15: 1197-1208.

11. Sperling RA, Pellagrino T, Li JK, Chang WH, Parak WJ. Electrophoretic Separation of Nanoparticles with Discrete Number of Functional Groups. Adv. Funct. Mater. 2006; 16: 943-948.

12. Jadzinsky PD, Calero G, Ackerson CJ, Bushnell DA, Kornberg RD. Structure of a Thiol Monolayer-Protected Gold
Nanoparticle at $1.1 \AA$ A Resolution. Science. 2007; 318: 430-433.

13. Yang J, Eom K, Lim E, Park J, Kang Y, Yoon DS, Na S, Koh EK, Suh J, Huh Y, Kwon TY, Haam S. In Situ Detection of Live Cancer Cells by Using Bioprobes Based on $\mathrm{Au}$ Nanoparticles. Langmuir. 2008; 24: 12112-12115.

14. Gibson JD, Khanal BP, Zubarev ER. Paclitaxel-Functionalized Gold Nanoparticles. J. Am. Chem. Soc. 2007; 129: 11653-11661.

15. Cao YC, Jin R, Mirkin CA. Nanoparticles with Raman Spectroscopic Fingerprints for DNA and RNA Detection. Science. 2007; 297: 1536-1540.

16. Zhou Y, Wang S, Zang K, Jiang X. Visual Detection of Copper (II) by Azide- and Alkyne-Functionalized Gold Nanoparticles Using Click Chemistry. Angew. Chem. Int. Ed. 2008; 47: 74547456.

17. Bowman M, Ballard TE, Ackerson C, Feldheim DL, Margolis DM, Malender C. Inhibition of HIV Fusion with Multivalent Gold Nanoparticles. J. Am. Chem. Soc. 2008; 130: 6896-6897.

\section{How to cite this article:}

M. Deepa, M. Surekha, V. Thushara Bindu, Deepti, K. Bhargavi and S. Deva sree. Gold nanoparticles in cancer therapy. Int. J. Adv. Pharm. Biotech., 2015; 1(2): 19-24. 